WALTER TROCKEL

dell'Università di Bielefeld (Germania Federale)

\title{
MARKET DEMAND BY NON-CONVEX PREFERENCES
}

(Conferenza tenuta il 7 aprile 1987)

\begin{abstract}
Sunto. - In questo lavoro si presenta una rassegna di studi sulla domanda di mercato.

Si studiano le condizioni sotto le quali tale domanda è una funzione continua dei prezzi e le condizioni che ne implicano la differenziabilità con continuità.
\end{abstract}

In a competitive equilibrium individual economic agents' decisions are decentralized by the price mechanism in such a way that they are compatible and therefore yield market clearing. A necessary assumption for this is the unique determinateness of the aggregate decisions by the prevailing equilibrium price system. Since nonconvexities of preferences cannot reasonably be precluded, individual demand at a given price system in general fails to be uniquely determined. This leads to the question, under which conditions the aggregate demand of a consumption sector is uniquely determined at every price system and hence, by the upper hemi-continuity of demand relations, is a continuous function of prices.

Since, however, even continuous functions can be unboundedly steep, continuity cannot prevent the demand to react extremely sensitive to price variations. To be sure, therefore, that slight price modifications do not lead to considerable variations in demanded quantities, and, hence, to significant deviations from equilibrium, one needs proportionality of price and demand quantities close to equilibria. This so called Lipschitz property is enjoyed, in particular, by continuously differentiable demand functions.

Continuous differentiability of the aggregate demand function is, moreover, an indispensable basic assumption in the theory of regular economies. For the relevance of regular economies I refer to DIERKER [1]. 
So we are confronted with the following two problems: under which conditions is market demand

1. uniquely determined, hence a continuous function of prices?

2. a continuously differentiable function of prices?

Already A. Cournot [2] knew that individual demand in general cannot be continuous. The continuity of market demand which he postulated is, according to CoURNOT, caused by the large number of economic agents, whise consumption characteristics are so different that the multi-valued demands sets, which are possible for all agents at some prices, are suitably distributed over all prices. COURNOT, however, even ascribed the Lipschitz property to continuous functions. This is not so surprising, since the true relation between continuity and differentiability was explored only 30 years later. Therefore, both of our problems date back to CoRUNOT. For him, however, the market demand was the starting point for the analysis.

But in a microeconomic framework in which market demand is derived via aggregation from individual demand, the problem is whether structural assumptions on the aggregate demand relation are compatible with economic behavioral assumptions for individual consumers.

The problem of unique determinateness of market demand was formulated in the early Seventies by HILDENBRAND [3] and DEBREU [4]. HILDENBRAND expressed the possibility that on the basis of a large number of different consumers classes of distributions of consumption characteristics might be identifiable for which market demand is uniquely determined. DEBREU sharpened this conjecture by replacing continuity of market demand by continuous differentiability.

Conceptual and formal difficulties prevented a fast progress in solving these problems. And soon it became obvious that the $C^{1}$ problem is a much deeper one than the $C^{0}$ one, the latter being treatable in principle also in a non-differentiable framework.

A question of fundamental significance for both problems was, how to formalize suitably the idea of sufficiently dispersed preferences. Differences in the way this question was tried to be answered resulted in different methodological approaches by which the problems were attacked. Uniqueness of market demand already re- 
quires the set of consumers with non-unique demand at a certain price system to be negligible. Accordingly, a suitable model needed the infinity of agents. Yet, if all of these happen to have identical characteristics, then individual and aggregate demand coincide. Therefore, one needs not only many, but also sufficiently diverse consumers.

A natural candidate for the formalization of suitable dispersion in Euclidean spaces in the Lebesgue measure. Unfortunately, the space of preferences does not provide enough structure allowing for a measure with comparable invariance properties. Due to this fact SONDERMANN [5] treated the aggregation problem for such sets of preferences, which can be parametrized by open subsets of Euclidean spaces. In this situation one concentrates only on an extremely thin subset of the whole space, but Lebesgue measure is available in principle. Clearly, the equal distribution represented by Lebesgue measure can be of use only, if this parametrized family of preferences represents a sufficiently « rich» demand behavior. Would, for example, the same non-convex preference be associated with every parameter, then even Lebesgue measure could not possibly induce an aggregate demand function.

In order to get a family of suitably dispersed preferences, SoNDERMANN [5] used a so called transversality condition. This is a differential topological assumption in the setup of preferences, which can be represented by continuously differentiable utility functions.

The effect of this condition is illustrated in figures 1 and 2 .



Fig. 1. - Rotation of indifference curves of a preference.

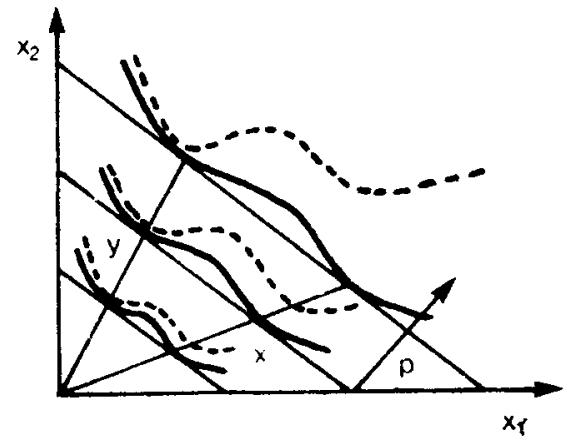

Fig. 2. - Rotation of preferences. 
In figure 1 a preference is drawn whose indifference curves are rotated in such a way that non-unique demand behavior at a given price system does occur only in a single income situation. But even if, as in figure 2, the given preference leads to two commodity bundles in the demand set for a whole interval of incomes, nonunique demand behavior remains still an exception, provided the parametrized family of preferences contains sufficiently many rotated neighboring preferences. Infinitesimal versions of those kinds of diversification, illustrated in the figures, are guaranteed by the transversality condition. Formally we get this conditions as follows:

Let $P$ be the positive orthant of the commodity space $\mathbb{R}^{l}$. Let $S=\left\{p \in P \mid p=\left(p_{1}, \ldots, p_{l-1}, 1\right)\right\}$ be the space of normalized prices. The set $A$ of consumers is assumed to be an open subset of $\mathbb{R}^{n}$. By $U^{r}$ we denote the set of $r$-times continuously differentiable $\left(C^{r}\right.$ for short) utility functions $u: P \rightarrow \mathbb{R}^{l}(r \geq 2)$, such that for all $x \in P$ we have

i) $D u(x) \gg 0 \quad\left(C^{r}\right.$-monotonicity)

ii) $u^{-1}(u(x))$ is closed in $\mathbb{R}^{2}$. (boundary condition)

«》» means bigger in all components.

Let $y$ be a Lebesgue continuous probability on the set $\mathcal{B}(A)$ of the Borel subsets of $A$. Let $E: A \rightarrow P \times U^{r}: a \mapsto\left(e_{a}, u_{a}\right)$ be a measurable mapping with $\int e_{a} v(d a) \ll \infty$, satisfying certain differentiability assumptions. Then the pair $(E, \nu)$ describes an economy.

The demand of consumer $a$ at the price system $p$ is then the set of best elements in his budget set

$$
\varphi(a, p)=\left\{x \in P \mid p x \leq p e_{a}, u_{a}(y)>u_{a}(x) \Longrightarrow p y>p e_{a}\right\} .
$$

The mean demand of the economy $(E, v)$ at the price system $p$ then is

$$
F_{v}(p)=\int_{A} \varphi(a, p) \nu(d a)
$$

Let $v: A \times(0, \infty) \times P^{2} \rightarrow \mathbb{R}$ be defined by

$$
v(a, t, x, y)=u_{a}(t x)-u_{a}(t y) .
$$


Then the transversality condition is

$$
\text { For all } x, y \in P, x \neq y \quad D v(., ., x, y) \neq 0 \text {. }
$$

The following result was established by SonDERMaNN [5] using methods of catastrophe theory.

Let $\nu$ be a Lebesgue-continuous probability on $(A, \mathcal{B}(A))$.

Let $E$ be such that $(T C)$ is fulfilled. Then $F_{y}: S \rightarrow P$ is a continuous function.

This result was generalized by W. HiLdENBRAND [6] to a nondifferentiable setup.

This parametric approach resulted in a positive answer as to uniqueness and continuity of aggregate demand. The differentiability problem remained unsolved. Moreover, no answer was given to the question, whether not only specially selected parametrized finite-dimensional subsets of the space of preferences, but also the whole space itself is endogenously endowed with sufficient diversification as to induce a unique and continuous mean demand.

These open problems led to a new non-parametric approach developped by E. and H. DierkeR and W. Trockel in $[7,8,9]$. This approach starts out from the idea that preferences are not the only consumption characteristics. A consumer is, in addition, depending on the specific context, described by his initial endowment with commodities or his initial wealth. In any case this can be described by an element in an Euclidean space. Also its dispersion can be described by means of the Lebesgue measure. Therefore, one can split aggregation into two steps, 1 . integration over wealth for fixed preferences, 2. integration over preferences. So a smoothing effect may be hoped for, even before one has to deal with dispersion of preferences. A first test of this procedure, whose proper goal is a differentiable demand, is to try to get uniqueness and continuity by this method. Indeed, this is possible. Let us consider the utility functions in $U^{r}$.

For $u \in U^{r}$ we define the associated normal map $g_{u}$ by

$$
g_{u}: P \rightarrow S: x \mapsto g_{u}(x)=D u(x) /(D u(x))_{l} .
$$


For $r=\infty$ we consider now the set of functions $u$ in $U^{r}$, satisfying

i) ( $C^{r}$-monotonicity) and ii) (boundary condition) and, in addition, the following rank assumption iii) $g_{u}$ has rank $l-1$.

For the space of those function with the $C^{\infty}$-Whitney topology E. and H. DIERKER and W. TROCKEL [7] proved the following result:

Given suitable wealth dispersion - the wealth distribution has to be Lebesgue-continuous - every preference with a utility representation outside a certain meager set in $U^{\infty}$ induces a demand relation aggregated over wealth $\Phi(\gtrsim, \cdot)$, which is a continuous function of prices.

When aggregation over the one-dimensional wealth space already leads to a continuous demand function for most preferences, how does the situation look like with the differentiability problem? There three different phenomena may possibly occur which can prevent the differentiability of demand.

First, there is a vanishing Gaussian curvature $\mathcal{X}_{Z}(x)$ in a point $x$ of an indifference surface of the preference $z$. The local flatness of the indifference surface caused by this vanishing Gaussian curvature leads to non-differentiatibility of the demand function, even for strict convex preferences (cf. DEBREU [4]).

Using wealth dispersion and methods of elementary catastrophe theory $\mathrm{E}$. and $\mathrm{H}$. DIERKER and W. TROCKEL [8] could prove the following: the demand function aggregated over wealth for a preference relation with $\mathcal{X}_{Z}\left(x_{0}\right)=0$ in the unique demanded commodity bundle $x_{0}$ at the price system $p_{0} \in S$ is continuously differentiable $\left(C^{1}\right)$ at the price system $p_{0}$. By this means one of the three possible disturbing effects was already «smoothed away» before aggregation over preferences took place. The remaining disturbing effects are demand sets with more than two commodity bundles and demand sets with two different commodity bundles $x, y$ with identical marginal utility vectors $D u(x)=D u(y)$. Also with regard to these phenomena there is a significant smoothing effect by integration over wealth by fixed preferences. Here only preferences are used whose utility representations are elements of $U^{\infty}$ and fulfil i), ii), iii). For mainly measure-theoretic reasons, instead of the 
Whitney-topology, the topology of $C^{\infty}$ uniform convergence on compact sets is chosen. The corresponding space of preferences is denoted $\mathscr{P}^{\infty}$. His topology can be characterized as well as the $C^{\infty}$ compact open topology on the corresponding normal functions $g_{\gtrsim}: P \rightarrow S, \gtrsim \in \mathcal{P}^{\infty}$. E. and H. DIERKER, and W. TROCKFl proved in this framework the following fact: For any given preference $\gtrsim \in \mathcal{P}^{\infty}$ there exist a nullset $N_{\gtrless}$ of the price space $S$ in such a way that the restriction to $S \backslash N_{\gtrsim}$ of the demand $\Phi(\gtrsim, \cdot)$ integrated over wealth is a continuously differentiable function. Such an «almost everywhere continuously differentiable " demand can have indefinitely steep slopes and is therefore, in general, not a Lipschitz function. Hence, for prices in $N_{\gtrsim} \Phi\left(z_{,}\right)$need not be uniquely determined.

The method of splitting aggregation into two steps turned out to be successful. The first step of integration over wealth already yielded considerable smoothing effects. Moreover, the work related to the first step of aggregation gave valuable hints how to proceed in the second step of aggregation. The problem now was to suitably formalize the concept of dispersion of preferences. It was supposed that a dispersion of only those aspects of preferences would suffice, which are important for the demand behavior, that means, which can be described in some way by prices and wealth. It was the aim that after integration over wealth the pathological demand behavior would in some sense be uniformly distributed over prices in such a way that similar demand behavior in similar price situations should occur with similar frequency.

In order to make these ideas clear, we consider the following actions of the space $S$ (a group under coordinatewise multiplication) onto itself, onto $P$, and onto $\mathcal{P}$. The space $\mathcal{P}$ can be $\mathcal{P}^{\infty}$ or any space of weakly monotone preferences. Let

$$
\begin{aligned}
& q=\left(q_{1}, \ldots, q_{l-1}, 1\right), p=\left(p_{1}, \ldots, p_{l-1}, 1\right), x=\left(x_{1}, \ldots, x_{l}\right) . \\
& S \times S \rightarrow S:(q, p) \mapsto q \circ p=\left(q_{1} p_{1}, \ldots, q_{l-1} p_{l-1}, 1\right) \\
& S \times P \rightarrow P:(q, x) \mapsto q \circ x=x^{q}=\left(q_{1} x_{1}, \ldots, q_{l-1} x_{l-1}, x_{l}\right) \\
& S \times \mathcal{P} \rightarrow \mathcal{P}:(q, \gtrsim) \rightarrow \geq_{q}
\end{aligned}
$$


Here $\gtrsim_{q}$ is defined by $\left[x \gtrsim y \Longleftrightarrow x^{q} \gtrsim_{q} y^{q}\right]$. The effect of these actions is illustrated in figure 3 .

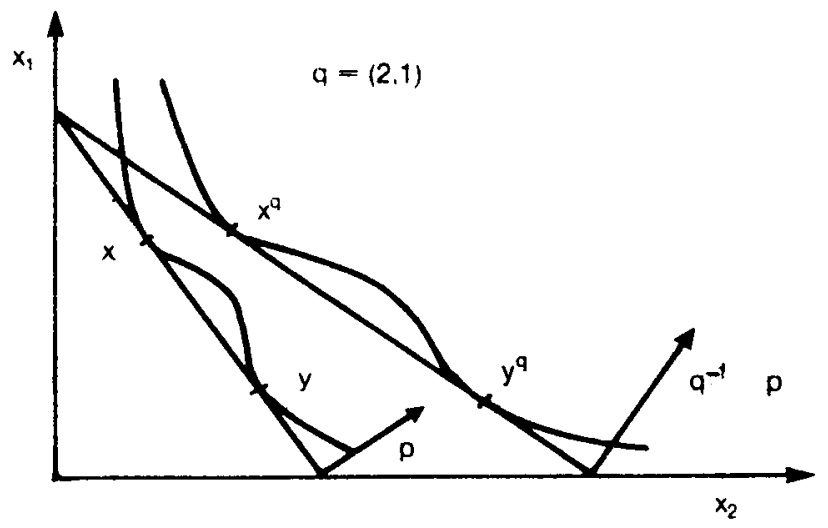

Fig. 3. - Effects of the action $q$ on prices, commodity bundles, and indifference curves.

For the individual demand relation $\varphi$ we get $q \circ \varphi(\gtrsim, w, p)=\varphi\left(\gtrsim_{q}, w, q^{-1} \circ p\right)$ with $q^{-1}=\left(q_{1}^{-1}, \ldots, q_{l-1}^{1}, 1\right)$

This can be written as well as

$$
\text { (*) } q \circ \varphi(\gtrsim, w, q \circ p)=\varphi\left(\gtrsim_{q}, w, p\right) \text {. }
$$

The set $[\gtrsim]=\left\{\gtrsim_{q} \mid q \in S\right\}$ denotes the orbit in $\mathcal{P}^{\infty}$ through $\gtrsim$ which is generated when $q$ runs through $S$. Now we can again split the integration of demand over $\mathcal{P}_{\infty}$ into two steps. First, one integrates within each orbit and after that «cross》 to the orbits. The first step is the essential one. Rather than integrating demand over all preferences $\gtrsim_{q}, q \in S$ of an orbit at price $p$, one can integrate the demand of the preference $\gtrsim$ over all prices $q^{-1} \circ p, q, \in S$. For this the Lebesgues-measure is available. By this means one can transport any Lebesgue-continuous probability on $S$ to each of the orbits $[\gtrsim], \gtrsim \in \mathcal{P}^{\infty}$. On this basis E. and H. DIFRKER and W. TrocKEL [9] were able to formalize price dispersion of preferences. The similarity to the ideas of CoURNOT sketched above is apparent. 


\section{Since}

$$
w=p \varphi(\gtrsim, w, p)=p \circ q^{-1} q \circ \varphi(\gtrsim, w, p)=q^{-1} \cdot p \varphi\left(\gtrsim_{q}, w, q^{-1} \circ p\right)
$$

holds true, the wealth remains invariant under these transformations. Therefore it is possible to perform separately aggregation over wealth and over preferences.

However, to get a continuously differentiable market demand measure-theoretical distribution assumptions do not suffice. In addition, one needs non-trivial methods of the singularity theory. Under the assumptions of wealth dispersion and price dispersion of preferences, and under additional compactness assumptions, and additional stability assumptions, which are partially difficult to interpret, one can prove that mean demand is a continuously differentiable function of prices. The corresponding theorem proved by E. and H. DIERKER and W. TrockEL [9] is the only existing result giving conditions under which market demand is continuously differentiable. Although most likely this theorem can be simplified and generalized on the basis of a deep understanding of the methods of the singularity theory, it seems quite sure that merely measuretheoretical distribution assumptions without additional stability assumptions do not suffice. Insofar DEBREU's conjecture could only partially be confirmed.

HILDEBRAND's uniqueness conjecture, however, can be completely confirmed by this approach also in the non-differentiable framework. It is well-known that also for non-convex preferences the individual demand is unique at almost all prices (cf. W. Trockel [10]). Therefore one can use the formula $\left({ }^{*}\right)$ above, to derive that for a given price system almost all preferences in an orbit induce a unique demand at almost all wealth situations.

Then only distribution assumptions are needed, namely wealth dispersion and price dispersion of preferences, to get a continuous market demand function (W. TROCKEL [11]). Since also local versions of such assumptions suffice, this means that also in case of non-convex preferences the market demand is generally uniquely determined unless an «oversharp observation» of individual consumption characteristics is performed.

A careful analysis of the problems sketched in this paper as well as a list of references can be found in W. Trockel [12]. 
SUMmary. - In this paper a survey is presented of works on market demand.

Conditions are examined under which such demand is a continuous function of prices and conditions which ensure its continuous differentiability.

\section{LITERATUR}

[1] E. DIERKer: Regular Economies, in K. J. Arrow, M. D. Intrilligator: Handbook of Mathematical Economics, Bd. 2, North Holland, Amsterdam 1982 , S. $795-830$.

[2] A. Cournot: Recherches sur les principes mathématiques de la théorie des richesses, Librairie des sciences politiques et sociales. M. Rivière \& Cie, Paris 1838.

[3] W. Hildobrand: Core and Equilibria of a large Economy, Princeton University Press, Princeton 1974.

[4] G. Debreu: Smooth Preferences, Econometrica 40 (1972) 603-615.

[5] D. SondermanN: Smoothing Demand by Aggregation, J. Math. Econ. 2 (1975) 201-224.

[6] W. Hildidrand: On the uniqueness of Mean Demand for Dispersed Families of Preferences, Econometrica 48 (1980) 1708-1710.

[7] E. Dinrkre, H. Dikrker, W. Trockor: Continuous Mean Demand Functions Derived from Non-Convex Preferences, J. Math. Econ. 7 (1980) $27-33$.

[8] E. Dierker, H. Dierker, W. Trockel: Smoothing Demand by Aggregation with respect to Wealth, J. Math. Econ. 7 (1980) 227-247.

[9] E. Dierker, H. DierkkR, W. Trockgr: Price-dispersed Preferences and $\mathbf{C}^{1}$ Mean Demand, J. Math. Econ. 13 (1984) 11-42.

[10] W. Trockrs: On the Uniqueness of Individual Demand at almost every Price System, J. Econ. Theory 38 (1984) 377-\$99.

[11] W. Trocker: Market Demand is a Continuous Function of Prices, Economics Letters 12 (1983) 141-146.

[12] W. Trockel: Market Demand. An Analysis of Large Economies with Non-Convex Preferences, Springer Verlag, Berlin 1984. 